\title{
Production of monozygotic mouse twins from microsurgically bisected morulae
}

\author{
H. Nagashima, K. Matsui, T. Sawasaki and Y. Kano \\ Stock Farm, Faculty of Agriculture, The University of Tokyo, Iwama, Ibaraki 319-02, Japan
}

\begin{abstract}
Summary. Mouse monozygotic twins were produced by bisection of the compacted morulae and transfer of the pairs of half-embryos after culture in vitro. The compacted morulae (about 16 cells) were microsurgically bisected, using a fine glass needle attached to a micromanipulator, without any supporting micro-instruments, after pretreatment for zona-softening and decompaction. About $80 \%$ of the morulae were bisected without visible cell damage. After $20 \mathrm{~h}$ in culture, the half-embryos were classified morphologically as eu-blastocysts, pseudo-blastocysts, or trophectodermal vesicles or non-integrated forms. After culture of 131 pairs of bisected morulae, 75 $(57 \cdot 3 \%)$ pairs of eu-blastocysts, $20(15.3 \%)$ pairs comprising a eu-blastocyst and pseudoblastocyst, and $9(6.9 \%)$ pairs of pseudo-blastocysts, were obtained. The pseudoblastocysts were considered to be derived from half-morulae in which some blastomeres were destroyed or dissociated as a result of micromanipulation. From 30 pairs of eu-blastocysts transferred to 21 recipients, 5 twin fetuses on Day 17 (18 pairs/9 recipients) and 3 twin male young (12 pairs/12 recipients) were obtained. Survival rate of the twin-embryo pairs was $27.8 \%$ at autopsy and $25.0 \%$ at term. None of the 20 pairs of pseudo-blastocysts transferred to 10 recipients gave rise to normal conceptuses.
\end{abstract}

\section{Introduction}

The artificial production of monozygotic multiple offspring by micromanipulation of early embryos has been extensively studied in mammals. Monozygotic sheep twins and cow triplets have been produced from totipotent blastomeres isolated microsurgically from 2-8-cell embryos (Willadsen, 1979, 1980; Willadsen \& Polge, 1981). These experiments involved agar embedding and culture of the 'blastomere-embryos' in an intermediate recipient. To simplify the culture procedure, it would be advantageous to obtain monozygotic sets of 'partial-embryos' from morulae or blastocysts, the preferred stages for transfer to recipient uteri. However, it has been reported that 'half-embryos' isolated from embryos after the 8-cell stage and subsequently cultured in vitro for a relatively short period could develop normally after transfer in rats (Nagashima \& Ogawa, 1981), and into twins in mice (Moustafa \& Hahn, 1978) and in cows (Williams, Elsden, \& Seidel, 1982; Ozil, Heyman \& Renard, 1982).

In the present study, mouse monozygotic twins were produced by bisecting compacted morulae, using a simple method devised by us, and transferring the pairs of half-embryos after a period of culture in vitro. The factors which may affect development of the halved morulae, i.e. the procedure for bisection and in-vitro culture of the bisected embryos, were investigated in an attempt to improve viability after transfer. 


\section{Materials and Methods}

Animals and embryos

In all experiments, embryo donors were the ICR strain of mice which were induced to superovulate (Fowler \& Edwards, 1957) and mated with male mice of the same strain or of the C57BL/6N strain. Consequently, these embryos were of the albino or pigmented type, respectively. Compacted morula-stage embryos (about 16 cells) were collected from uteri of the donors $76 \mathrm{~h}$ after the hCG injection. Whittingham's culture medium (Whittingham, 1971) was used for collection of embryos. Recipients were also of the ICR strain and were mated with vasectomized males the day after donors were mated.

Bisection of morulae was performed following the method developed by Nagashima \& Ogawa (1981), with modification for mouse embryos. The zonae pellucidae of the embryos were digested mildly by incubation for $1.5-2.0 \mathrm{~min}$ in $1-2 \mathrm{ml}$ pronase solution $(0.5 \%$ in Dulbecco's PBS), followed by thorough washing in culture medium. The 'zona-softened' embryos were transferred to $0.4 \mathrm{ml}$ of $\mathrm{Ca}^{2+}-\mathrm{Mg}^{2+}$-free PBS (Dulbecco's PBS) covered with liquid paraffin after rinsing twice with $\mathrm{Ca}^{2+}-\mathrm{Mg}^{2+}$-free PBS, and incubated for $20 \mathrm{~min}$ at $37^{\circ} \mathrm{C}$ in the presence of $5 \% \mathrm{CO}_{2}$ in humidified air. Immediately decompaction was complete, as judged by the appearance of distinct cell outlines in the morulae (Pl. 1, Fig. 1), the embryos were placed in culture medium again.

Groups of 5-10 pre-treated embryos were placed in a small droplet of medium (Dulbecco's PBS $+20 \%$ calf serum) on a microscope slide at room temperature. Microsurgery was carried out at $\times 100$ using an Olympus (CK) inverted microscope with the aid of phase-contrast illumination. A fine glass needle attached to a micromanipulator (Narishige, MO-102) was used to bisect embryos. No supporting micro-instruments, such as an 'egg-holder' pipette, were needed. The tip of the needle (corresponding to a cutting edge; approximately $15 \mu \mathrm{m}$ in diameter), adjusted to give an angle of about $30^{\circ}$ to the surface of the slide, was placed over the embryo in an equatorial plane. By lowering the needle slowly, the embryo could be bisected. In this way, the blastomere mass and the zona were divided into two approximately equal fragments (P1. 1, Fig. 2). As shown in Pl. 1, Fig. 2 , in most cases, each cluster of the separated blastomeres remained in its bowl-shaped hemispherical zona after the manipulation.

\section{PLATE 1}

Fig. 1. Mouse morulae (about 16 cells) after decompaction. $\times 200$.

Fig. 2. A monozygotic pair of half-morulae immediately after bisection. In this specimen, each half contains about 10 intact blastomeres. $\times 200$.

Fig. 3. A monozygotic pair of half-morulae, with a destroyed blastomere in one half (arrowed). $\times 200$.

Fig. 4. A monozygotic pair of eu-blastocysts developed from a bisected morula, after $20 \mathrm{~h}$ in culture. Note the distinct inner cell mass and well-developed trophectoderm. $\times 200$.

Fig. 5. A monozygotic pair of pseudo-blastocysts, after $20 \mathrm{~h}$ in culture; Note a small clump of cells $(\nabla)$ enclosed by the trophectoderm, and debris (arrowed) from destroyed blastomeres. $\times 200$.

Fig. 6. A monozygotic pair comprising a trophectodermal vesicle (left) and non-integrated form (right), after $20 \mathrm{~h}$ in culture. $\times 200$.

\section{PLATE 2}

Fig. 7. A monozygotic pair of expanding eu-blastocysts at the time of transfer, after $33 \mathrm{~h}$ in culture. $\times 200$.

Fig. 8. Intact control blastocysts at the same developmental stage as in Fig. $4 . \times 200$.

Fig. 9. Intact control expanding blastocysts at the same developmental stage as in Fig. 7 . $\times 200$. 
PLATE 1

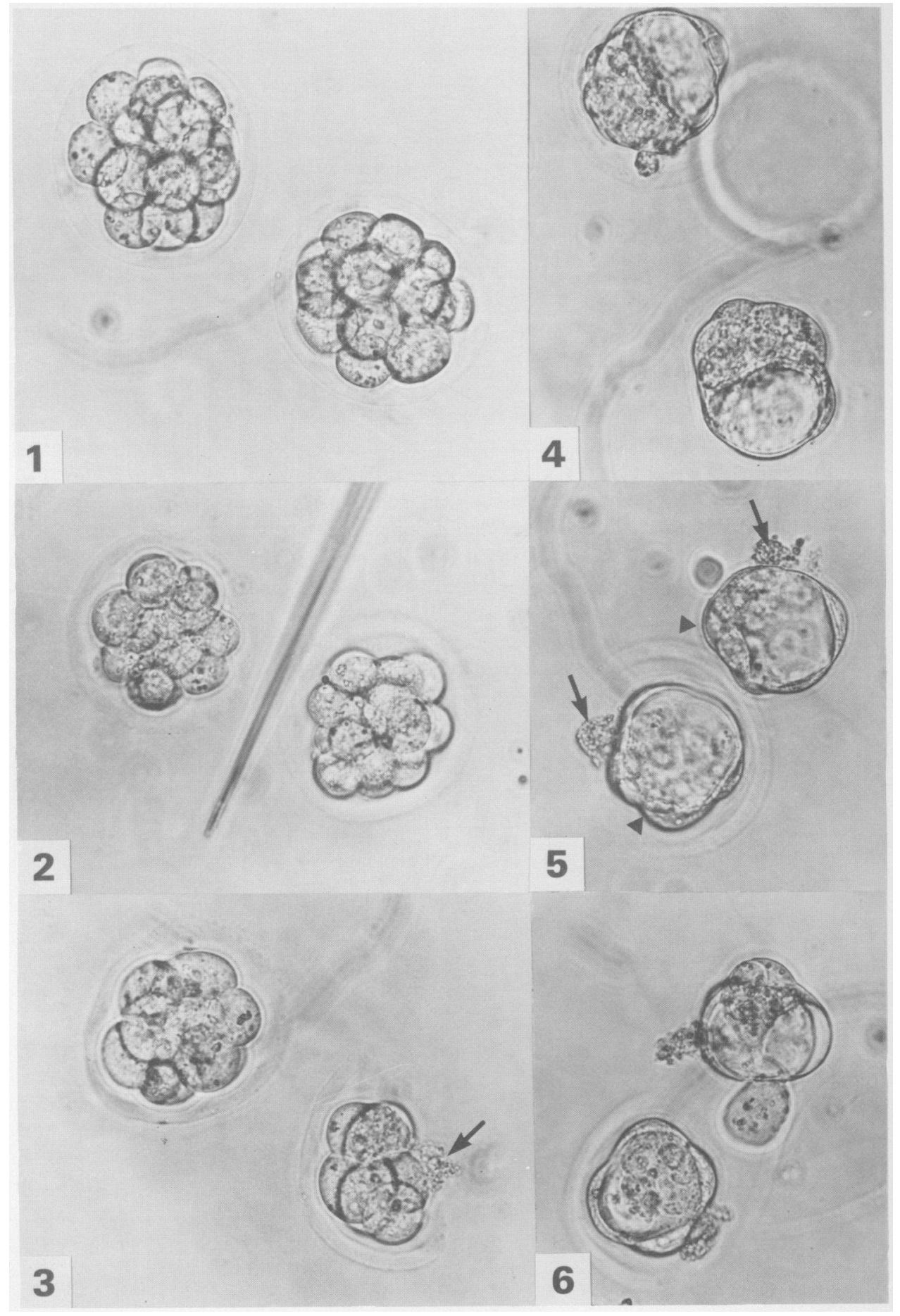




\section{PLATE 2}

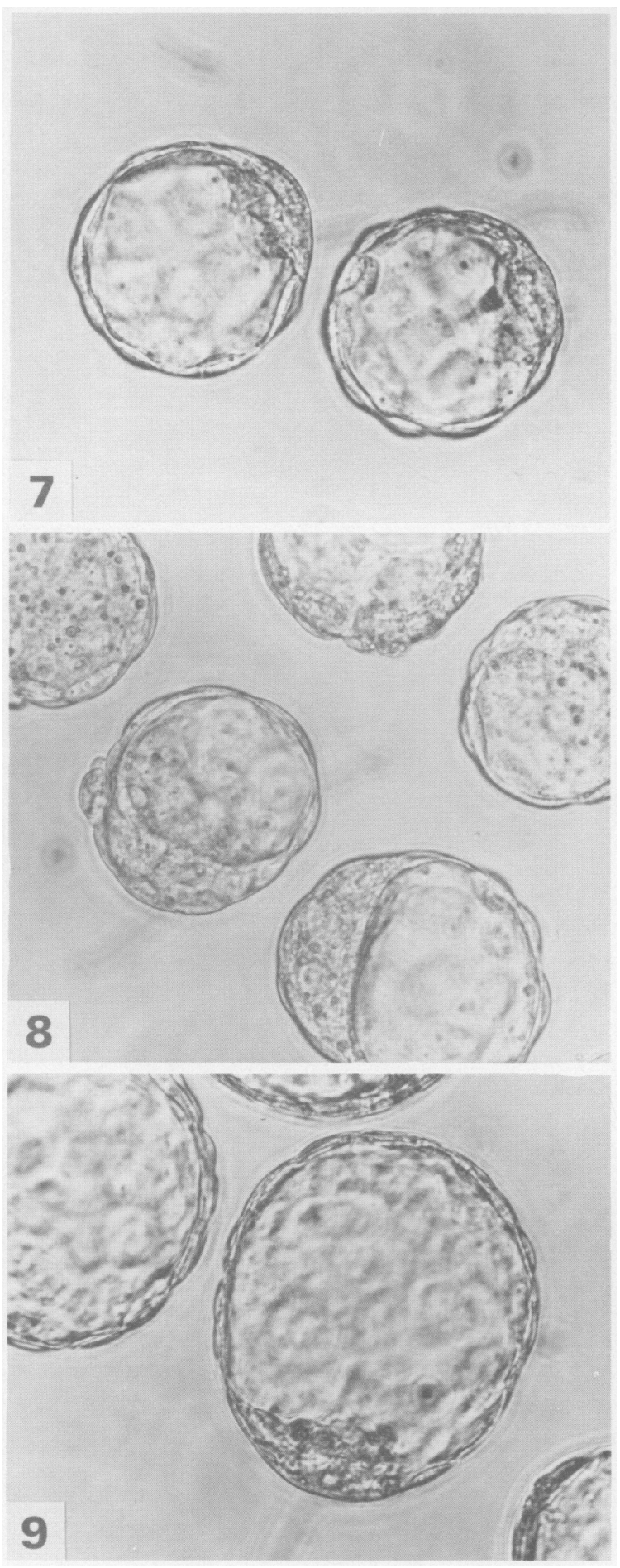


After bisection, each pair of the half-morulae was transferred to a small droplet of Whittingham's medium (Whittingham, 1971) covered with liquid paraffin in a Petri dish. The bisected embryos were handled gently, so that little of the separated blastomere mass escaped from the open, cut zona. Intact morulae which had undergone zona-softening treatment were cultured as controls. The culture was performed in a humidified atmosphere of $5 \% \mathrm{CO}_{2}$ in air at $37^{\circ} \mathrm{C}$ for $33-$ $36 \mathrm{~h}$. All the embryos were examined with an inverted microscope and phase-contrast illumination and their developmental state was recorded.

\section{Transfer of half-embryos}

After in-vitro culture for $33-36 \mathrm{~h}$, the bisected morulae were transferred to pseudopregnant recipients on the morning of Day 4 (Day 1 being the day copulation plug was present). The halfembryos which developed from the bisected morulae were classified morphologically into the following three groups after culture for $20 \mathrm{~h}$. (1) Eu-blastocysts were those containing a distinct inner cell mass and well-developed trophectoderm, although smaller than normal control blastocysts (Pl. 1, Fig. 4 ; Pl. 2, Fig. 8). (2) Pseudo-blastocysts appeared to contain a small clump of cells enclosed by the trophectoderm (poorly developed inner cell mass) and, in the majority of cases, debris from destroyed blastomeres or dissociated blastomeres excluded from the trophectodermal layer (Pl. 1, Fig. 5). (3) Trophectodermal vesicles, i.e. trophectoderm with no enclosed cells and non-integrated forms which were disorganized clusters of cells, some of which were vacuolated (Pl. 1, Fig. 6). The pairs of half-embryos which were classified as 'eu-blastocysts' and 'pseudoblastocysts', i.e. both halves of the pair being of the same type, were transferred after additional culture for 13-16 h. These embryos were in the expanding stage at transfer (Pl. 2, Fig. 7). The developmental ability of the pseudo-blastocysts was compared with that of the eu-blastocysts.

Recipients were anaesthetized with pentobarbitone sodium (Nembutal: Abbott Laboratories), and the ovary and the tubal end of the uterine horn on each side were exteriorized in turn through a dorsolateral incision in the abdominal musculature. The tubal end of the horn was punctured with a 25-gauge hypodermic needle and the embryos were deposited in the lumen with a transfer pipette. A monozygotic pair of half-embryos were transferred into each horn of the recipient. Autopsy of recipients was performed on Day 17 of pregnancy and the numbers of fetuses or resorptions in each horn were recorded. The presence of 2 fetuses in a single horn was the criterion for twinning.

To obtain live births, a pair of eu-blastocysts (albino) with 3-9 intact control embryos (pigmented; Pl. 2, Fig. 9) were transferred. At delivery, live young were recorded and examined externally for abnormalities. Stillborn young and cannibalism were also recorded, when possible.

\section{Results}

\section{Microsurgical bisection of morulae}

Of 158 morulae $127(80.4 \%)$ were considered to be bisected without visible cell damage (Pl. 1, Fig. 2). Bisection of the other 31 embryos resulted in obvious destruction $(15,9 \cdot 5 \%)$ or dissociation $(16,10 \cdot 1 \%)$ of blastomeres in one or both halves (Pl. 1, Fig. 3). Destruction occurred mainly in the blastomeres in the path of the microneedle. When decompaction of the embryos was incomplete, it was difficult to bisect them without injuring the blastomeres.

\section{In-vitro development of the bisected morulae}

A total of 131 morulae were bisected and all the monozygotic pairs of half-embryos produced were cultured. The half-morulae underwent recompaction and blastulation in culture after 4-6 and $12-18 \mathrm{~h}$ respectively, although their development was retarded compared with that of intact control 
morulae. The half-embryos were examined microscopically at $20 \mathrm{~h}$ in culture and morphologically classified into eu-blastocysts, pseudo-blastocysts and trophectodermal or non-integrated forms.

After culture for $20 \mathrm{~h}$ the 131 bisected morulae produced a total of $75(57.3 \%)$ monozygotic pairs of eu-blastocysts, $20(15 \cdot 3 \%)$ pairs comprising a eu-blastocyst and pseudo-blastocyst, $9(6.9 \%)$ pairs of pseudo-blastocysts, and $27(20.6 \%)$ pairs which included a trophectodermal vesicle, a nonintegrated form, or a degenerated embryo.

\section{Development of blastocysts after transfer}

Experiment 1: development assessed by autopsy. The results for monozygotic pairs of eublastocysts transferred to 9 recipients are shown in Table 1 . In the pregnant recipients there were 2-3 fetuses/recipient. None of the 20 pairs of pseudo-blastocysts which were transferred to 10 recipients gave rise to normal conceptuses. At autopsy on Day 17, 5 recipients that had received 20 embryos revealed only 11 sites of decidual reaction.

Table 1. Development of monozygotic (MZ) pairs of mouse eu-blastocysts after transfer

\begin{tabular}{|c|c|c|c|c|c|c|}
\hline & $\begin{array}{c}\text { No. of } \\
\text { half-embryos } \\
\text { transferred }\end{array}$ & $\begin{array}{l}\text { No. of } \\
\text { MZ pairs } \\
\text { transferred }\end{array}$ & $\begin{array}{l}\text { No. of } \\
\text { recipients }\end{array}$ & $\begin{array}{l}\text { No. of } \\
\text { live fetuses } \\
\text { or young }\end{array}$ & $\begin{array}{l}\text { No. of } \\
\text { resorptions, } \\
\text { still births or } \\
\text { cannibalized } \\
\text { young }\end{array}$ & $\begin{array}{l}\text { No. of } \\
\text { pairs of } \\
\text { MZ twins }\end{array}$ \\
\hline $\begin{array}{l}\text { Exp. } 1 \text { (assessed by autopsy } \\
\text { on Day 17) } \\
\text { Exp. } 2 \text { (assessed at term) }\end{array}$ & $\begin{array}{l}36 \\
24\end{array}$ & $\begin{array}{l}18 \\
12\end{array}$ & $\begin{array}{c}7 / 9 \\
(77 \cdot 8 \%) \\
12 / 12 \\
(100 \%)\end{array}$ & $\begin{array}{c}15 \\
(41 \cdot 7 \%) \\
10 \\
(41 \cdot 7 \%)\end{array}$ & $\begin{array}{c}5 \\
(13 \cdot 9 \%) \\
3 \\
(12 \cdot 5 \%)\end{array}$ & $\begin{array}{c}5 \\
(27 \cdot 8 \%) \\
3 \\
(25.0 \%)\end{array}$ \\
\hline & & & \multicolumn{4}{|c|}{$\begin{array}{c}13 \\
(54 \cdot 2 \%)\end{array}$} \\
\hline
\end{tabular}

Experiment 2: development assessed at term. All the monozygotic pairs of eu-blastocysts (albino) transferred with 67 intact control embryos (pigmented) led to pregnant recipients which gave birth to $10\left(7 \sigma^{x} \sigma^{*}, 1\right.$ \& 2 unexamined) albino and 29 pigmented living offspring on Day 20-22 (Table 1). The 3 sets of monozygotic twins were all males.

\section{Discussion}

In early mouse development, the morula stage is regarded as the initiating stage of differentiation of blastomeres (Ducibella, 1977; Johnson, Handyside \& Braude, 1977; Johnson, 1979). During compaction of the morula some blastomeres become completely enclosed by others. These enclosed 'inner blastomeres' and the surrounding 'outer blastomeres' are thought to differentiate into inner cell mass (ICM) and trophectoderm in the blastocyst, respectively (Tarkowski \& Wróblewska, 1967; Hillman, Sherman \& Graham, 1972). Spatial interaction between the component blastomeres in the compacted morula appears to play an important role in prescribing the developmental fate of the blastomeres (Johnson, 1981; Handyside, 1980).

In this experiment, the developmental fate of individual blastomeres as precursors of the ICM and trophectoderm must have been upset by decompacting and bisecting the compacted morula. It was, however, observed that the half-morulae underwent re-compaction 4-6 h after the manipulation and subsequently developed into blastocysts. During this re-compaction, blastomeres of half-morulae are presumably reorganized as precursors of ICM and trophectoderm, permitting the formation of viable half-blastocysts (eu-blastocysts). 
Tarkowski \& Wróblewska (1967) investigated the in-vitro development of blastomeres isolated from 4- and 8-cell mouse embryos. They suggested that reduction of blastomere number to a level at which a small number of 'inner blastomeres' was present at the late morula stage would result in a blastocyst with a small ICM. In the development of our halved morulae which had less than half the normal number of blastomeres because some blastomeres were destroyed or dissociated by the micromanipulation, regeneration of the ICM precursors might be incomplete. As Tarkowski \& Wróblewska (1967) described, lack of the precursor blastomeres must have resulted in a poorly developed ICM which had no ability to form an entire fetus. Damaged half-morulae were therefore unlikely to form viable half-blastocysts: these pseudo-blastocysts, in which damage was limited to a few blastomeres, were, in fact, unable to give rise to normal conceptuses, although they did induce decidual reactions.

These results for the pseudo-blastocysts, showing that viability of the half-morulae was seriously affected by a slight loss of the component blastomeres, indicate that it is important to know how much damage can be tolerated. The seriousness of the damage must depend on the number and kind $(1 / 8,1 / 16$, or $1 / 32$ blastomeres) of blastomeres lost. Unfortunately, it was usually difficult to know how many and what kind of blastomeres had been destroyed or dissociated by the micromanipulation. In addition, not all the half-morulae which had been observed as having no sign of cell damage immediately after the bisection developed into eu-blastocysts. The production rate of the viable twin-blastocysts was $57 \cdot 3 \%$, although the expected success rate for bisection was $80.4 \%$. Accordingly, it is also difficult to predict the developmental ability of the half-morulae at the time of micromanipulation. This study showed that only the 'eu-blastocysts' could give rise to normal conceptuses, and so selection after in-vitro culture for a limited time is considered advantageous. Selective transfer of the micromanipulated embryos predicted to be viable would be particularly important when the number of available recipients is limited.

The distinct feature of our method was that the bisected embryos remained in the halved zonae at least at the beginning of the culture period. The hemispherical zonae thus remaining might facilitate the re-compaction of the embryos and, further, might support their development into the viable blastocysts, although Moustafa \& Hahn (1978) have reported that the half-embryos separated from mouse 8-16-cell embryos could develop normally in culture with or without the zonae. In our experiments in freezing preservation of the rat half-morulae (Nagashima \& Ogawa, 1981), the component blastomeres, which had aggregated compactly, were often dissociated after freezing and thawing. Consequently, only $6 \%$ of the frozen-thawed half-morulae developed into blastocysts of normal shape in culture, although $78.1 \%$ of the unfrozen embryos developed normally. It was thought that if the half-morulae were protected by a surrounding zona pellucida, the blastomeres would not dissociate so readily, and the viability of the embryos would not be fatally affected. We therefore suggest that, to produce mouse twins efficiently, the morulae must be bisected without damaging any blastomeres, and the blastomeres of the half-morulae must be kept undissociated during the culture period before the transfer.

\section{References}

Ducibella, T. (1977) Surface changes of the developing trophoblast cell. In Development in Mammals, Vol. 1, pp. 5-30. Ed. M. H. Johnson. North-Holland, Amsterdam.

Fowler, R.E. \& Edwards, R.G. (1957) Induction of superovulation and pregnancy in mature mice by gonadotrophins. J. Endocr. 15, 374-384.

Handyside, A.H. (1980) Distribution of antibody- and lectin-binding sites on dissociated blastomeres from mouse morulae : evidence for polarization at compaction. J. Embryol. exp. Morph. 60, 99-116.
Hillman, N., Sherman, M.I. \& Graham, C. (1972) The effect of spatial arrangement on cell determination during mouse development. J. Embryol. exp. Morph. 28, 263-278.

Johnson, M.H. (1979) Intrinsic and extrinsic factors in preimplantation development. J. Reprod. Fert. 55, $255-265$.

Johnson, M.H. (1981) Membrane events associated with generation of a blastocyst. In Membrane Research, pp. 1-38. Ed. A. L. Muggleton-Harris. Academic Press, New York.

Downloaded from Bioscientifica.com at 04/26/2023 $04: 59: 34 \mathrm{AM}$ via free access 
Johnson, M.H., Handyside, A.H. \& Braude, P.R. (1977) Control mechanisms in early mammalian development. In Development in Mammals, Vol. 2, pp. 67-97. Ed. M. H. Johnson. North-Holland, Amsterdam.

Moustafa, L.A. \& Hahn, J. (1978) Experimentelle Erzeugung von identischen Mäusezwillinge. Dt. tierärztl. Wschr. 85, 242-244.

Nagashima, H. \& Ogawa, S. (1981) Studies on the developmental potential and the survival after the deep freezing of microsurgically dichotomized morula embryos in rats and rabbits. Japan. J. Anim. Reprod. 27, 12-19.

Ozil, J.P., Heyman, Y. \& Renard, J.P. (1982) Production of monozygotic twins by micromanipulation and cervical transfer in the cow. Vet. Rec. 110, 126-127.

Tarkowski, A.K. \& Wróblewska, J. (1967) Development of blastomeres of mouse eggs isolated at 4- and 8-cell stage. J. Embryol. exp. Morph. 18, 155-180.

Whittingham, D.G. (1971) Culture of mouse ova. $J$. Reprod. Fert., Suppl. 14, 7-21.

Willadsen, S.M. (1979) A method for culture of micromanipulated sheep embryos and its use to produce monozygotic twins. Nature, Lond. 277, 298-300.

Willadsen, S.M. (1980) The viability of early cleavage stages containing half the normal number of blastomeres in the sheep. J. Reprod. Fert. 59, 357-362.

Willadsen, S.M. \& Polge, C. (1981) Attempts to produce monozygotic quadruplets in cattle by blastomere separation. Vet. Rec. 108, 211-213.

Williams, T.J., Elsden, R.P. \& Seidel, C.E., Jr (1982) Identical twin bovine pregnancies derived from bisected embryos. Theriogenology 17, 114, Abstr.

Received 25 August 1983 